\title{
EL ROL DE LA SOCIEDAD CIVIL EN EL RECONOCIMIENTO DE LA SEGURIDAD ALIMENTARIA COMO BIEN PÚBLICO REGIONAL EN LATINOAMÉRICA
}

\section{THE ROLE OF CIVIL SOCIETY IN RECOGNITION OF FOOD SECURITY AS A REGIONAL PUBLIC GOOD IN LATIN AMERICA}

Susana Herrero Olarte

\begin{abstract}
RESUMEN
La seguridad alimentaria puede tratarse como un bien público regional, como en el caso de los EE.UU. o la UE, cuando los titulares de obligaciones así lo consideren, o cuando los titulares de derechos les obliguen al reclamarlos como tales. La demanda sólo podrá darse cuando la sociedad civil los reconozca como tales, siempre y cuando cuente con suficiente información. El análisis de fuentes primarias y secundarias permite concluir que en el caso latinoamericano los movimientos sociales de afectados, y las ONG nacionales y regionales, y la ciudadanía en general reclaman mejoras en el ámbito de la seguridad alimentaria, pero todavía a escala nacional por un desconocimiento claro de las oportunidades de la consideración de la seguridad alimentaria como bien público regional.
\end{abstract}

\section{PALABRAS CLAVE}

Globalización; regionalización; Latinoamérica; Bien Público Regional; seguridad alimentaria

\begin{abstract}
Food security can be treated as a regional public good, as in the case of EU or US when debenture holders so wish, or when right holders oblige them to claim them as such. The demand can only occur when the civil society to recognize them as such, as long as sufficient information. The analysis of primary and secondary sources to the conclusion that in the case of Latin America social movements affected, and national and regional NGOs and the public are demanding improvements in the area of food security but still nationally by clear disregard opportunities for the consideration of food as a regional public security.
\end{abstract}

\section{KEY WORDS}

Globalization; regionalization; Latin America; A regional public good; food security 


\section{INTRODUCCIÓN}

La desnutrición continúa en el siglo XXI siendo un reto en Latinoamérica. No sólo es un problema en sí, sino que además limita el potencial de desarrollo de la región por las externalidades que genera la falta de seguridad alimentaria. Es preciso identificar la estrategia que logre erradicar la desnutrición de manera viable y sostenible. En el mundo hay varios países y regiones que ya lo han logrado. Si se pretende realizar la comparación con Latinoamérica, es preciso analizar el caso de los EE.UU. y la UE por su similitud en tamaño, población y variedad de países y Estados.

Los EE.UU. y la UE desarrollaron unas políticas agrícolas que perseguían erradicar la desnutrición de su ciudadanía, mejorar la calidad de vida de sus agricultores, y aumentar el saldo positivo de la balanza de pagos de los alimentos. Lograron lo que se propusieron con una estrategia marcada por la transferencia de recursos hacia la producción de alimentos de su región, el límite legal a las importaciones y la apertura de los mercados internacionales para que aceptaran su producción subvencionada.

Es un ejemplo claro, en ambos casos, de soberanía alimentaria y del tratamiento de los alimentos como bienes públicos regionales. Los bienes públicos regionales son un tipo de bienes públicos globales. Son los bienes públicos que no pueden proveerse desde un punto de vista nacional, afectados por causas que no se originan en los países, y que generan externalidades que afectan a un conjunto de países que comparten frontera.

A nivel latinoamericano se reconoce además tanto a nivel nacional, como regional y por los organismos internacionales relacionados la necesidad de considerar los alimentos como bienes públicos regionales. No obstante, en la práctica no se desarrollan como tales ni por los titulares de obligaciones ni de derecho. En efecto, por el lado de la oferta los resultados de la UNASUR para desarrollar políticas que en la práctica supongan la provisión de alimentos en la región son muy limitados. En cuanto a la demanda, esta es débil y está poco desarrollada, lo que invita a pensar en un desconocimiento estructural de los bienes públicos regionales en general, y del tratamiento de los alimentos como tales en particular.

En este trabajo nos concentraremos en los titulares de derecho y en la pobre demanda de los alimentos como bienes públicos regionales en Latinoamérica, que consideramos 
obedece a un débil conocimiento de la población sobre el tema, lo que implica que, necesariamente, no los pueden reclamar. Para valorar el conocimiento de la ciudadanía, se estudia el comportamiento de tres colectivos seleccionados durante el siglo XXI. Los conjuntos seleccionados son los grupos regionales de presión, los movimientos sociales afectados por la desnutrición y la sociedad civil en general. En el primer y en segundo caso, se estudian sus manifiestos públicos a través de sus declaraciones, publicaciones y entrevistas para ver si en algún caso aparece alguna declaración relacionada con la consideración de los alimentos como bienes públicos regionales. En el tercero, se valora, a través de encuestas, su conocimiento sobre el resultado que la integración podría tener en la seguridad alimentaria nacional y regional.

Los resultados muestran cómo existe una demanda de alimentos como bienes públicos pero hacia el Estado, desconociéndose la responsabilidad regional y global y, especialmente, el potencial que tendría la intervención más allá del ámbito nacional.

En cualquier caso es preciso identificar cómo mejorar la demanda, lo que necesariamente pasa por lograr que la ciudadanía mejore sus niveles de información para que tome las mejores decisiones y genere la demanda suficiente, que tenga que satisfacerse mediante la oferta.

\section{Los ALIMEntos como Bienes Públicos Regionales (BPR)}

La desnutrición afecta negativamente la capacidad para generar ingresos (Baldwin y Weisbrod, 1974) y a la productividad laboral (OMS, 2003), además de la capacidad de entendimiento, y al desarrollo cognitivo (Jukes et al. 2002). Genera también enfermedades neuronales, dermatológicas, circulatorias y estomacales (Latham, 2002), así como una menor esperanza de vida y un mayor número de muertes infantiles, según la FAO (2008). La desnutrición limita entonces de manera directa e indirecta el crecimiento y el desarrollo porque parte de la ciudadanía tiene afectado su capacidad para la mejora, condicionando la sostenibilidad de los cambios en el medio y largo plazo.

América Latina ha sabido traducir el crecimiento de su PIB en una reducción de la desnutrición del 46\% de 1992 a 2014, superando el promedio del mundo en 25 puntos para el mismo periodo. Además, la volatilidad ha sido sustancialmente menor (Banco 

Año XXIII, N³6/Junio 2017, ISSN 2468-9912. DNDA: 5318259 en línea

Susana Herrero Olarte El rol de la sociedad civil en el reconocimiento de la seguridad alimentaria como bien público regional en Latinoamérica Págs. 67-85

Mundial, 2016). No obstante, en 2014 todavía el 11\% de la población estaba en condición de subnutrición (FAO, 2016), lo que limita su capacidad para crecer y desarrollarse con todo su potencial, generando cambios estructurales.

En varios países y regiones se ha alcanzado la seguridad alimentaria, pero merece la pena atender al trabajo realizado por los Estados Unidos y la Unión Europea porque como América Latina, tienen Estados muy distintos entre sí y cuentan con una superficie y una población similares, lo que permite pensar en analizar sus estrategias, para utilizarlas después en el diseño de políticas públicas latinoamericanas para erradicar la desnutrición. El Departamento de Agricultura (USDA) de los EE.UU. nació en 1892 y la Política Agraria Común (PAC) de la UE en 1957. Sus objetivos eran la autosuficiencia como región para protegerse en el caso de conflicto futuro, garantizar la alimentación de la población, mejorar la calidad de vida de los agricultores y garantizar una balanza comercial agropecuaria positiva

La alimentación de la población se aseguraba garantizando que la cantidad de comida producida podía abastecer a la población y que era lo suficientemente barata para comprarse. Hasta 2003, la producción per cápita de la UE y los EE.UU. estuvo por encima de la media mundial, llegándose a desperdiciar 179 kilos de comida por persona al año en la década de los noventa (BIO Intelligence Service, 2012). En cuanto al precio de los alimentos, el gasto de las familias no ha dejado de descender desde que se tienen registros. En el caso europeo, el porcentaje del gasto en alimentos sobre el total de los ingresos por familia eran, en 1988 del 12,8\%, en 1994 del 10,4\%, en 1999 del 8,3\%, y en 2005 del 7,6\% (Economic Research Service, 2010). En los EE.UU. el gasto era en 1933 del $25,2 \%$, en 1950 del $22 \%$, en 1977 del $13,4 \%$, en 1993 del $11,1 \%$, y en 2013 del $9,8 \%$ (Rural Development USDA, 2010).

En cuanto a la mejora de la calidad de vida de los agricultores, los ingresos por familia en la UE en 2011 eran de 16.670 dólares al año (Comisión Europea, 2014), mientras que en los EE.UU. eran de 16.000 (Asociación de Trabajadores Agrícolas de Carolina del Norte (2007), muy por encima del promedio mundial.

En tanto a la balanza comercial de los alimentos, el resultado ha sido especialmente bueno en los EE.UU., que han tenido siempre una balanza positiva, mientras en Europa el 

Año XXIII, N³6/Junio 2017, ISSN 2468-9912. DNDA: 5318259 en línea

Susana Herrero Olarte El rol de la sociedad civil en el reconocimiento de la seguridad alimentaria como bien público regional en Latinoamérica Págs. 67-85

resultado per cápita de 1962 a 2014 resulta negativo, si bien se registran once años con signo positivo (Banco Mundial, 2016).

La política desarrollada en ambos casos tenía dos características comunes. Por un lado, los Estados miembros de ambos grupos supeditaban sus políticas a las de los actores supranacionales (FDA, 2010). Además, la política debía contar con el presupuesto necesario para desarrollarse y no adaptarse a los recursos disponibles en el momento. El presupuesto del USDA era el $0,004 \%$ del presupuesto de los EE.UU. Suponía 440 dólares por persona al año (CIA, 2015; USDA, 2015 y 2012b). El presupuesto de la PAC era el $42 \%$ del general de la UE y alcanzaba los 228 dólares por persona (UE, 2010; Dirección General de Agricultura y Desarrollo Rural de la Unión Europea, 2015).

Los instrumentos utilizados han sido, sobre todo al principio, las subvenciones a los productores que permitían la venta por debajo del coste de producción en todas sus versiones. Los países perjudicados por los alimentos subvencionados, con los que no podían competir ni nacional ni internacionalmente, se quejaban ante la Organización Mundial del Comercio (OMC) porque se contravenían sus principios fundamentales por muchas excepciones que se hubiesen hecho en el artículo 9. Tras la reacción de la OMC, presionada también por los grupos organizados de la sociedad civil, que recriminaba su actitud a los EE.UU. y la UE, éstos cambiaban la modalidad de la subvención: en especie y en efectivo a los agricultores, a los productores de insumos agropecuarios, a los consumidores, y/o a los exportadores (Herrero, 2015).

El empeño de los EE.UU. y la UE para conseguir los objetivos propuestos solo puede entenderse si los alimentos son bienes públicos, en este caso, Bienes Públicos Regionales (BPR) (Herrero, 2014).

Los BPR son un subgrupo de los Bienes Públicos Globales (BPG). Los BPG son bienes públicos puros o impuros que al no suministrarse generan una serie de externalidades negativas que afectan a varios Estados del mundo. No pueden proveerse desde el ámbito nacional, por lo que es necesario que se suministren desde el ámbito internacional (Kaul et al. 1999). La provisión no puede discriminar a ningún segmento de la población, ni a ninguna generación presente o futura (García-Verdugo Sales y Martín, 2004) y debe asegurarse por los titulares de obligaciones (Martens y Hain, 2001). La sociedad civil debe 
ser la que reclame su suministro o regulación (Martens y Hain, 2008), siempre y cuando cuente con la suficiente información (Herrero, 2015). Ejemplos de bienes públicos globales son el agua del mar o el aire, que por mucho que un país quiera preservarlos en buen estado, si los otros países no quieren no se podrá alcanzar el objetivo de conservación.

La seguridad alimentaria se reconoce como BPG ya desde el trabajo de Kaul et al. (1999). Las causas de la inseguridad alimentaria en un país tienen cada vez más un origen no nacional. Además, si la ciudadanía de un país no cuenta con los niveles suficientes de seguridad alimentaria se afecta negativamente la población del resto del mundo por considerarse el acceso a los alimentos un derecho humano, pudiendo además ser el estado que genere conflictos y enfermedades que alcancen a otros países. Las autoridades deberían asegurar su provisión sin discriminaciones ni comprometer las posibilidades de la ciudadanía futura. Además, los titulares de derechos, si están bien informados, deberían reclamar que los alimentos se traten como BPG.

Los principales organismos internacionales relacionados, como el Banco Mundial, la FAO, el PMA y el FIDA, reconocen la consideración de los alimentos como BPG a través de sus organismos de investigación especializada como la Asociación Mundial de Seguridad Alimentaria (GFSP) (GFSCI, 2013) o el Comité de Seguridad Alimentaria Mundial (CFS) (Page, 2013).

Desde la academia, Ravi (2002) llamaba a la regionalización o globalización como la manera de conseguir un mejor suministro y acceso a los alimentos. Unnevehr (2004, 2007) hacía referencia a los fallos de mercado, que de tratarse adecuadamente, pueden apoyar una mejor provisión de los alimentos siendo estos bienes públicos globales. Ausín (2010) Ilamaba la atención sobre la necesidad de definir los alimentos como bienes públicos globales, y proveerlos y suministrarlos como tales desde las autoridades competentes. Gifra y Beltrán (2012) incidían en el reconocimiento de los alimentos como BPG desde la legalidad.

La acotación regional limita la afectación a un grupo de países de la misma zona, lo que permite plantear políticas que necesiten de menos actores para alcanzar acuerdos, que deberán ser además menos ambiciosas porque tendrán que cubrir las necesidades de 
Revista Aportes para la Integración Latinoamericana Año XXIII, N³6/Junio 2017, ISSN 2468-9912. DNDA: 5318259 en línea

Susana Herrero Olarte El rol de la sociedad civil en el reconocimiento de la seguridad alimentaria como bien público regional en Latinoamérica Págs. 67-85

menos personas ambiciosas. Al considerar el caso latinoamericano, sería más adecuado pensar en los alimentos como en BPR en lugar de BPG.

\section{EL TRATAMIENTO DE LOS ALIMENTOS EN LATINOAMÉRICA COMO BPR}

En la práctica, desde los organismos internacionales la consideración de los alimentos en Latinoamérica como BPR se ha traducido en varios proyectos concretos. El Instituto Interamericano para la Cooperación en Agricultura (IICA) ha financiado varias investigaciones sobre el tema (Piñeiro, 2009 y Sepúlveda, 2009), que profundizaban especialmente en los acuerdos que deberían seguirse (IICA, 2001). El Banco Interamericano de Desarrollo (BID) ha invertido 90 millones dólares a través de 114 acciones desarrolladas en marco de la Iniciativa de Bienes Públicos Regionales (IBPR) implementando seis proyectos con un presupuesto de 3,5 millones (BID, 2015).

En el ámbito político, y desde y para la región, se vienen alcanzando acuerdos entre grupos de países desde la década de los setenta. En 1977, México y Venezuela firmaron el primer tratado para la creación del Sistema Económico Latinoamericano (SELA) para protegerse ante embargos o restricciones al comercio de alimentos, y generar el embrión de un mecanismo de integración y cooperación regional, de una manera parecida a la creación de la UE. En 1983, se creó el Comité de Acción sobre Seguridad Alimentaria Regional (CASAR), a raíz del conflicto de las Malvinas, del que participaron Argentina y Reino Unido. Argentina y Uruguay impulsaron el Tratado de Asistencia Regional para Emergencias Alimentarias (TAREA) en 1986, aunque entró en vigor a principios de los noventa. Buscaban eliminar la influencia extranjera que condicionaba su seguridad alimentaria, generar un mecanismo de compra para cuando hubiese una emergencia y tener además una provisión para contingencias. En 2011, entró en vigor el Tratado Constitutivo de la Unión Nacional del Sur (UNASUR), firmado en 2008, suscrito por Argentina, Bolivia, Brasil, Colombia, Ecuador, Guayana, Paraguay, Perú, Suriname, Uruguay y Venezuela. El Grupo de Trabajo sobre Seguridad Alimentaria y Lucha contra la Malnutrición, con sede en Uruguay, era el marco de trabajo para desarrollar acciones en el marco de la alimentación regional (UNASUR, 2015). Las prioridades definidas fueron el diseño y la implementación de los Sistemas de Seguridad Alimentaria y Nutricional y los Programas de Acceso a los Alimentos y Agricultura Familiar apoyándose en la 
cooperación con el resto del mundo, tratando de manera transversal de preservar el acceso al agua, y preservando los ecosistemas relacionados con la seguridad alimentaria. En la práctica, las acciones llevadas a la práctica han sido las desarrolladas por la UNASUR, concretadas en alcanzar el consenso en torno a la definición de seguridad alimentaria nacional y socializar experiencias positivas nacionales y entre grupos de países (Vázquez, 2013, 2014).

Las pocas medidas desarrolladas invitan a preguntarse por las causas que se relacionan con la oferta o con la demanda de los alimentos como BPR en Latinoamérica.

\section{LA DEMANDA DE LOS ALIMENTOS COMO BPR EN LATINOAMÉRICA}

La oferta de BPG, cuyo estudio se ha desarrollado mucho más que la demanda, presenta dificultades desde el origen en la medida en que la cuestión no está clara ni consensuada en tanto a los bienes públicos nacionales, lo que supone un reto al considerar los globales y regionales.

El análisis de la oferta de BPG nació antes incluso de que estos se denominaran como tales. Ya en la década de los sesenta se planteaba cómo lograr proveer ciertos bienes que de manera nacional era imposible suministrar (Olson y Zeckhauser, 1966). A continuación se profundizó sobre cómo debe ser la relación entre países para trabajar de manera conjunta y lograr proveer de ciertos bienes a sus naciones (Sandler et al. 1978). La cooperación se erigió como la forma en la que debían relacionarse los países (Keohane, 1984), si bien al no alcanzarse los resultados previstos, cobró fuerza en los noventa la idea de un organismo supranacional capaz de proveerlos (Desai, 2003).

Todavía la cuestión del actor que debe proveer los BPG no está resuelta ni en la teoría ni en la práctica, con lo que siguen sin proveerse adecuadamente. Entre las causas que responden a la relajada voluntad con la que se abordan este tipo de temas destacan la falta de prioridad en la agenda política nacional y el cuestionamiento general que necesariamente se haría de los organismos intemacionales que por el momento se han estado encargando de los BPG, como las Naciones Unidas.

En cuanto a la demanda, el principal reto de que exista y se desarrolle adecuadamente es que la ciudadanía sepa que los BPG son el instrumento capaz de atender cuestiones que 

Año XXIII, N³6/Junio 2017, ISSN 2468-9912. DNDA: 5318259 en línea

Susana Herrero Olarte El rol de la sociedad civil en el reconocimiento de la seguridad alimentaria como bien público regional en Latinoamérica Págs. 67-85

a nivel nacional no pueden tratarse, como la calidad del aire o del agua. Sólo así podrán reclamar a nivel nacional como sociedad civil que se suministren bien a través de la cooperación, bien a través de un organismo supranacional responsable.

Los mismos retos de la oferta y la demanda de BPG se reconocen en su dimensión regional. En efecto, la desidia de los titulares de obligaciones y el desconocimiento de los titulares de derechos aparecen como problemas recurrentes también en la provisión efectiva de los alimentos como BPR.

Al considerar la cuestión de los alimentos como BPR en Latinoamérica por el lado de la oferta, se atiende a la falta de atención a la respuesta concreta y práctica de los grupos de integración relacionados para ofrecer las herramientas necesarias, cuestión que se invita a trabajar en los siguientes procesos de investigación.

En tanto al estudio de la demanda latinoamericana, cuestión que desarrolla el presente trabajo, es preciso analizar el reconocimiento de este tipo de bienes desde la sociedad civil para comprobar si podrían o no reclamarlos ante los titulares de obligaciones competentes a nivel regional. Para realizar el análisis es preciso considerar el conocimiento de tres grupos de ciudadanos con capacidades distintas de incidir en las políticas que desarrollen las autoridades. Por un lado los colectivos afectados pueden incidir, a través de sus manifestaciones y declaraciones en los medios de comunicación y redes sociales, en la ciudadanía que después tendrá que votar. Las ONG tienen la capacidad de movilizar la opinión de la sociedad civil a través de plataformas pensadas para la sensibilización y actuar en foros que generen propuestas de ley a nivel nacional y regional. Además, es preciso valorar el conocimiento que de partida presenta la ciudadanía de a pie en general y que incide con su voto en la toma de decisiones de los gobiernos nacionales y regionales.

\section{ENTENDIMIENTO DE LA SOCIEDAD CIVIL LATINOAMERICANA DE LOS ALIMENTOS COMO BPR}

Los movimientos sociales en Latinoamérica se han desarrollado en un contexto de desigualdad estructural y de lucha de clases, reclamando en definitiva una sociedad que dignifique a sus ciudadanos y caracterizándose por el enfrentamiento con el gobierno nacional, buscando y generando procesos de cambio rápidos y radicales. 
Hasta la década de los setenta destacaron los grandes movimientos de afectados que reclamaban mejoras en las condiciones salariales y laborales (Calderón \& Jelin, 1987). En la década de los ochenta, las grandes protestas dieron paso a movimientos sociales en forma de guerrilla en respuesta de los gobiernos militares o de extrema derecha del momento, buscando el cambio radical. Desde la década de los noventa, la falta de derechos sociales y las políticas neoliberales que habían dado lugar a la década perdida de los ochenta lograron aupar al gobierno a la nueva clase política de izquierdas en Venezuela, Argentina, Brasil o Bolivia. Fueron gobiernos más participativos y moderados que se pudieron acompañar de mejoras generalizadas de las condiciones económicas. La presión de los movimientos sociales se redujo y comenzó a concretarse en cuestiones menos generales y más particulares como son la gratuidad de la educación o la no violencia contra la mujer. Era el resultado de una masa crítica cada vez mejor formada, con más acceso a la información por el proceso de globalización, y de unos grupos mejor organizados que pudieron mejorar la calidad de las reivindicaciones, ofreciendo la sociedad civil soluciones, propuestas para el diálogo, etc. En la segunda década del siglo $\mathrm{XXI}$, la reducción de los presupuestos por el descenso en el precio de las materias primas y el desgaste de los gobiernos de izquierdas, que no cumplieron con las expectativas de parte de la ciudadanía ni erradicaron el nepotismo y la corrupción, han generado nuevas protestas en la región.

De manera paralela al resto de movimientos, las revueltas campesinas se han ido dando en toda la región. Tradicionalmente ignorados desde las autoridades de gobierno, los agricultores se han caracterizado por perpetuar, generación tras generación, las peores condiciones de vida y el menor acceso a los derechos humanos de la región. Los niveles de pobreza superaban en todos los casos la media nacional y presionaban a la baja, junto a la falta de servicios públicos, los indicadores nutricionales, de salud y educación. Los principales reclamos de los movimientos campesinos han sido por la falta de acceso a factores de producción como la tierra o el agua, el reconocimiento de sus derechos sociales y culturales, y por amenazas relacionadas con el proceso de globalización, como son los transgénicos, o los tratados comerciales.

En todos los casos, salvo contadas excepciones, los movimientos sociales agrarios en Latinoamérica asignan la responsabilidad de su situación al gobierno nacional y hacia él 

Año XXIII, N³6/Junio 2017, ISSN 2468-9912. DNDA: 5318259 en línea

Susana Herrero Olarte El rol de la sociedad civil en el reconocimiento de la seguridad alimentaria como bien público regional en Latinoamérica Págs. 67-85

se dirigen. Bien sea porque no cuentan con los bienes y derechos a nivel nacional o porque se sienten amenazados a nivel regional o global, los titulares de obligaciones designados son los Estados, lo que invita a pensar en una falta de información al respecto.

El avance del proceso de globalización también ha servido para, en el ámbito de la seguridad alimentaria, generar cada vez más alianzas entre Organizaciones No Gubernamentales (ONG). El papel de las ONG en la internacionalización de las cuestiones relacionadas con la alimentación en Latinoamérica ha sido fundamental. Por un lado, se han apoyado en la legislación relacionada con los derechos humanos para contar con herramientas que ante los titulares de obligaciones, las legitimaran en sus reclamos para generar procesos de cambio. Además, se han relacionado con otras organizaciones y organismos de la región y del mundo para visibilizar retos todavía por alcanzar y financiar procesos para revertir condiciones de desnutrición en casi todos los países de la región. Como resultado, se han creado algunas organizaciones que trabajan a nivel latinoamericano, como el Instituto para el Desarrollo Rural de Sudamérica (IPDRS) una iniciativa con sede en Bolivia para promover enlaces, sinergias y acciones de desarrollo rural de base campesina indígena en la región sudamericana; o la Sociedad Científica Latinoamericana de Agroecología (SOCLA), que promueve desde Perú la investigación y el conocimiento de la agroecología en la región.

No obstante, la experiencia general es la conformación de plataformas de ONG de distintos países de la región relacionadas con la seguridad alimentaria que suman esfuerzos e intercambian experiencias. En la siguiente tabla se detalla la información de algunas de las que suman el mayor número de organizaciones, incluyendo cuántas organizaciones forman parte, de cuántos países, si reciben fondos de la cooperación al desarrollo y si se posicionan en su relación con el proceso de globalización o regionalización. 
El rol de la sociedad civil en el reconocimiento de la seguridad alimentaria como bien público regional en

Tabla 1. Organizaciones regionales latinoamericanas sobre seguridad alimentaria

\begin{tabular}{l|c|c|c|c} 
& $\begin{array}{c}\text { Organizacion } \\
\text { es } \\
\text { participantes }\end{array}$ & Países & $\begin{array}{c}\text { Fondos de } \\
\text { cooperación }\end{array}$ & $\begin{array}{c}\text { Reclama } \\
\text { integración }\end{array}$ \\
\hline $\begin{array}{l}\text { International Land Coalition (ILC). } \\
\text { América Latina }\end{array}$ & 26 & 10 & Sí & No \\
\hline $\begin{array}{l}\text { Foro internacional andino amazónico } \\
\text { de desarrollo rural }\end{array}$ & 12 & 4 & No & So \\
\hline $\begin{array}{l}\text { Asociación Latinoamericana de } \\
\text { Organismos de Promoción (ALOP) }\end{array}$ & 9 & 5 & Sí & No \\
\hline $\begin{array}{l}\text { Red Latinoamericana de Comercio } \\
\text { Comunitario (RELACC) }\end{array}$ & 14 & 11 & No & Sí \\
\hline $\begin{array}{l}\text { Coordinadora Latinoamericana } \\
\text { de Organizaciones del Campo (CLOC) }\end{array}$ & 33 & 11 & No & Sí \\
\hline $\begin{array}{l}\text { Movimiento Agroecológico de América } \\
\text { Latina y El Caribe (MAELA) }\end{array}$ & 210 & 15 & No & Sí \\
\hline $\begin{array}{l}\text { Alianza por la Soberanía Alimentaria de } \\
\text { América Latina y el Caribe }\end{array}$ & 56 & 10 & & No
\end{tabular}

Fuente: Elaboración propia

Generalmente, y pese a su clara visión regional, las organizaciones ponen su atención en lo local, en la recuperación de lo tradicional, de lo hecho hasta entonces, que se limita por la acción de las empresas agrícolas. Desde un tono místico en general y reivindicativo en particular, la relación con lo internacional se limita a tratar de evitar el impacto negativo del proceso de globalización: "Paradójicamente entonces, el enorme avance tecnológico que vive la humanidad y el acceso a nuevos recursos abundantes en nuestra región, antes que una oportunidad para la equidad, la solidaridad y la justicia, pueden tornarse en una condena" (ALOP, 2005). Trata de impedirse el avance de las multinacionales de la alimentación, que se entienden como el enemigo: "Debemos derrotar el modelo agrícola impuesto por las corporaciones del agronegocio que apoyado por los capitales financieros internacionales" (CLOC, 2015) y de los transgénicos como MELA, que entre los indicadores de su Plan Estratégico 2011-2015 incluía: "Han aumentado las alianzas en 
cada país y a nivel internacional para incidir en normativas nacionales e internacionales respecto al derecho de los agricultores/as sobre los recursos genéticos; libre circulación de las semillas tradicionales; no al patentamiento y no a las semillas transgénicas." (MELA, 2011).

No obstante, si bien se aprecia un tímido y puntual reconocimiento positivo de los procesos de integración de los titulares de obligaciones "Reconociendo el avance de los procesos regionales y continentales de integración como UNASUR, ALBA, MERCOSUR y CELAC" (CLOC, 2015), todavía se contemplan como espacios marco de trabajo, en los que no tienen responsabilidades reales, que aún se atribuyen a los gobiernos nacionales.

La regionalización se entiende como una oportunidad de entendimiento, de intercambio de experiencias y de unión para reclamar ante las autoridades nacionales medidas que protejan a los productores y consumidores de las amenazas que presenta el proceso de globalización. No se valora la actuación como titulares de derechos ante titulares de obligaciones regionales, dado que no se contemplan las oportunidades que pueden generarse al considerar los alimentos como bienes públicos regionales.

La principal razón es el desconocimiento sobre el tema. En las 25 entrevistas mantenidas con organizaciones de cinco países de la región que tienen como objetivo mejorar las condiciones para alcanzar la seguridad o soberanía alimentaria, ninguno de los entrevistados sabía qué son los bienes públicos regionales ni había considerado que la política agrícola podría manejarse mejor desde la región que a nivel país. Los resultados son similares a los registrados al considerar la valoración de la ciudadanía en general.

Para valorar el apoyo de la ciudadanía a la integración regional, se atiende a los datos de Latinobarómetro fruto de casi 20.000 entrevistas en 18 países de la región. El $72 \%$ de los encuestados estaba en 2013 a favor de la integración latinoamericana económica, mientras el $60 \%$ apoyaba la integración también política (Corporación Latinobarómetro, 2015). La voluntad de la ciudadanía de contar con una mayor integración regional es pues indiscutible.

De cara a analizar en qué medida la población es más crítica con las políticas alimentarias nacionales y preferiría una gestión regional cuando tiene toda la información, se ha realizado un análisis con información primaria. Doscientas cincuenta encuestas se 

Año XXIII, N³6/Junio 2017, ISSN 2468-9912. DNDA: 5318259 en línea

Susana Herrero Olarte El rol de la sociedad civil en el reconocimiento de la seguridad alimentaria como bien público regional en Latinoamérica Págs. 67-85

realizaron en julio y agosto de 2015 en la ciudad de Quito, a dos grupos de 125 personas cada uno, al norte y sur de la ciudad. El Grupo 1 contaba con un $75 \%$ de personas que se consideraban a sí mismas de clase media alta o alta. El $91 \%$ contaba con estudios universitarios. En el Grupo 2, el $68 \%$ se definía como clase media baja o baja. El $48 \%$ no tenía estudios universitarios. En lo que respecta a las políticas de alimentos aplicadas en el país, el $73 \%$ considera que son mejorables o muy mejorables. En cuanto a las políticas de desarrollo rural la crítica aumenta hasta el 89\%. El Grupo 1 se muestra especialmente descontento, si bien la brecha con el Grupo 2 en el primer caso es de tres puntos y en el segundo de diez. El Grupo 1 tiene un mayor conocimiento de lo que es la UNASUR y considera que el futuro ecuatoriano será mejor en una América Latina unida. En tanto a si la política agrícola debería gestionarse desde la UNASUR se aprecian valores muy similares entre ambos grupos, pese a que el $42 \%$ de los encuestados del Grupo 2 no sabían lo que es la UNASUR. Esto se debe a que, al considerar en un $72 \%$ que la política nacional de alimentos no es la adecuada, que sea otro organismo en el que la desarrolle puede mejorarla.

Los resultados confirman la idea de que contra más información tiene una persona, más crítica es ante la política alimentaria y de desarrollo rural nacional y más defiende la impulsada a nivel regional.

\section{CONCLUSIONES}

En el caso de los bienes públicos globales, contar con la suficiente información es especialmente importante, sobre todo por el desconocimiento de la ciudadanía sobre el tema.

El proceso de globalización no puede desarrollarse de manera positiva y con todo su potencial sin considerar los bienes públicos globales, un elemento fundamental capaz de regular las amenazas y las oportunidades del propio proceso. No siempre los bienes públicos globales son la forma más adecuada, sino que pueden ser los bienes públicos regionales los que se gestionen y provean mejor. Es el caso de la seguridad alimentaria alcanzada en zonas como los EE.UU. y la UE a través de políticas supranacionales que han considerado a los alimentos como bienes públicos regionales. 

Año XXIII, N³6/Junio 2017, ISSN 2468-9912. DNDA: 5318259 en línea

Susana Herrero Olarte El rol de la sociedad civil en el reconocimiento de la seguridad alimentaria como bien público regional en Latinoamérica Págs. 67-85

En Latinoamérica, donde erradicar la desnutrición es todavía un reto, los alimentos todavía no se suministran como bienes públicos regionales ni desde el lado de la oferta ni desde la demanda. Por el lado de la oferta, no existen las organizaciones lo suficientemente empoderadas como para poder gestionar políticas regionales integrales en torno a la soberanía alimentaria ni parece que haya el interés desde los Estados. En cuanto a la demanda, ésta no es lo suficientemente fuerte como para presionar a los titulares de obligaciones para que generen la necesaria oferta a nivel regional.

El análisis en tres grupos de la sociedad civil que formarían parte de la potencial demanda de los alimentos como BPR muestra un desconocimiento generalizado de lo que son los BPG o los BPR. Su ejercicio como titulares de derecho se acota al ámbito nacional, sin considerar titulares de obligaciones regionales, salvo contadas excepciones. La regionalización se contempla como la manera de unir fuerzas para luchar contra los efectos negativos de la globalización, sin valorar las externalidades positivas que podrían potenciarse.

Es preciso entonces abordar la necesidad de informar a la sociedad civil sobre la consideración de los alimentos desde una visión supranacional y regional latinoamericana. El objetivo es doble, por un lado, que puedan ejercer como titulares de derechos y por el otro que puedan mejorar los bienes públicos globales y por ende el proceso de globalización, como parte intrínseca de él que son.

\section{BIBLIOGRAFÍA}

ALOP (2015). "Que es ALOP". Disponible: http://www.alop.org.mx/?q=iniciob (consulta realizada 12/01/2017).

ASOCIACIÓN DE TRABAJADORES AGRÍCOLAS DE CAROLINA DEL NORTE (2007). Salario por horas de los agricultores, Office of Rural Health and Community Care, Washington, Estados Unidos.

AUSíN Txetxu (2010). "El Derecho a Comer: Los Alimentos Como Bien Público Global", en: Arbor Ciencia, Pensamiento y Cultura, vol 186, № 745, Septiembre - Octubre, España. .

BALDWIN Robert. y WEISBROD Burton (1974). "Disease and labor productivity", en: Economic Development and Cultural Change, vol 22, № 3, Abril, Wiscosin, Estados Unidos. 
Revista Aportes para la Integración Latinoamericana Año XXIII, N³6/Junio 2017, ISSN 2468-9912. DNDA: 5318259 en línea

Susana Herrero Olarte

El rol de la sociedad civil en el reconocimiento de la seguridad alimentaria como bien público regional en

Latinoamérica

Págs. 67-85

BANCO INTERAMERICANO DE DESARROLLO (BID) (2015). "Acerca del BID". Disponible:http://www.iadb.org/es/acerca-de-nosotros/acerca-del-banco-interamericanode-desarrollo,5995.htm (consulta realizada 15/01/2017).

BANCO MUNDIAL (2016). "Base de datos". Disponible: http://datos.bancomundial.org/ (consulta realizada 02/12/2016).

BIO INTELLIGENCE SERVICE (2012). Preparatory Study on Food Waste Across EU 27, Bruselas, Bélgica

CALDERÓN Fernando y JELIN Elizabeth (1987). Clases y Movimientos Sociales en América Latina: Perspectivas y Realidades, Centro de Estudios de Estado y Sociedad, Buenos Aires, Argentina.

CIA (2015). "The World Factbook". Disponible: https://www.cia.gov/library/publications/theworld-factbook/fields/2056. html (consulta realizada 05/02/2017).

CLOC (2015). "Declaración final del IV Congreso de la CLOC". Disponible: http://www.cloc-viacampesina.net/index.php/es/temas-principales/pronunciamientos (consulta realizada 19/01/2017).

COMISIÓN EUROPEA (2014). "EU farm economics summary 2011", en: EU Agricultural and Farm Economics Briefs, № 1,Septiembre, Bruselas, Bélgica..

CORPORACIÓN LATINOBARÓMETRO (2015). "Opinión pública latinobarómetro, 19952015". Disponible: http://www10.iadb.org/intal/intalcdi/PE/2015/15817.pdf (consulta realizada 16/01/2017).

DESAI Meghnad (2003). "Public Goods: A Historical Perspective”, en: Kaul, I., Conceiçao, P. Le Goulven, K. y Mendoza, R. U. Providing Global Public Goods. Managing Globalization, Oxford University Press, New York and Oxford, Estados Unidos e Inglaterra.

DIRECCIÓN GENERAL DE AGRICULTURA Y DESARROLLO RURAL DE LA UNIÓN EUROPEA (2015). Agricultura y Desarrollo Europeo, Bruselas, Bélgica.

ECONOMIC RESEARCH SERVICE (2010). "Base de datos de la Unión Europea". Disponible: $h$ ttp://ec.europa.eu/eurostat/data/database (consulta realizada 04/02/2017).

FAO (2008). El estado mundial de la agricultura y la alimentación. Biocombustibles: perspectivas, riesgos y oportunidades, FAO, Roma, Italia.

FDA (2010). "Preguntas generales sobre la Administración de Medicamentos y Alimentos". $\quad$ Disponible: http://www.fda.gov/AboutFDA/Transparency/Basics/EnEspanol/ucm196466.htm (consulta realizada 25/01/2017).

GARCÍA - VERDUGO Javier y MARÍN José (2004). Bienes Públicos Globales, Política Económica y Globalización, Ariel, Barcelona, España.

GFSCI (2013). "Global Food Safety Curricula Initiative". Disponible: http://foodsafety.iufost.org/ (consulta realizada 19/01/2017). 
Revista Aportes para la Integración Latinoamericana Año XXIII, N³6/Junio 2017, ISSN 2468-9912. DNDA: 5318259 en línea

Susana Herrero Olarte El rol de la sociedad civil en el reconocimiento de la seguridad alimentaria como bien público regional en Latinoamérica

Págs. 67-85

GIFRA Julia y BELTRÁN Susana (2012). "El Derecho Humano a la Alimentación y al Agua".

Disponible: http://www.ieee.es/Galerias/fichero/cuadernos/CE_161_Seguridad_Alimentaria_y_Segurid ad_Global.pdf (consulta realizada 21/12/2016).

HERRERO Susana (2014). "La seguridad alimentaria como Bien Público Global", en: Comentario Internacional: revista del Centro Andino de Estudios Internacionales, 13. Disponible: $\quad$ http://repositorio.uasb.edu.ec/bitstream/10644/4146/1/12-OT-HerreroLa\%20seguridad.pdf (consulta realizada 14/02/2017).

(2015). "Análisis de la provisión nacional de alimentos en Sudamérica para su reconsideración como Bienes Públicos Regionales (BPR)" en: Ponencia en III Seminario Integración Latinoamericana. Disponible: http://www.momagri.org/UK/a-look-atthe-news/Agricultural-subsidies-A-telling-measure-of-the-changes-in-worldagriculture_1396.html (consulta realizada 14/02/2017).

JUKES Matthew, MCGUIRE Judith, METHOD Frank. y STERNBERG Robert (2002). "Nutrición y Educación". Disponible: http://www.unscn.org/files/Publications/Briefs_on_Nutrition/Brief2_SP.pdf (consulta realizada 19/01/2017).

KAUL Inge, GRUNBERG Isabelle y STERN Marc (1999). "Bienes Públicos Mundiales. Cooperación internacional en el siglo XXI", en: Oxford University Press, Nueva York y Oxford, Estados Unidos e Inglaterra..

KEOHANE Robert (1984). "After Hegemony: Cooperation and Discord in the World Political Economy", en: Princeton University Press, Nueva Jersey, Estados Unidos..

LATHAM Michael (2002). "Nutrición humana en el mundo en desarrollo". Disponible: http://www.fao.org/docrep/006/w0073s/w0073s00.htm\#Contents (consulta realiza 29/12/2016).

MARTENS J. y HAIN R. (2001). Bienes públicos globales, World Ecology, Economy and Development, Berlín, Alemania.

(2008). "Bienes públicos globales". Disponible: www.amycos.org/interactivos/pregunto/media/pdf/ficha9.pdf (consulta realizada 02/01/2017).

MELA (2011). "Plan Estratégico Maela 2011-2015". Disponible: http://maelaagroecologia.org/IMG/pdf/Plan_Estrategico_MAELA_2011-2015.pdf (consulta realizada 3/02/2017).

OLSON Mancur y ZECKHAUSER Richard (1966). "An Economic Theory of Alliances", en: Review of Economics and Statistics, $\mathrm{N}^{\circ} 48$, Octubre.

OMS (2003). "Salud, crecimiento económico y reducción de la pobreza", en: Informe del Grupo de Trabajo I de la Comisión sobre Macroeconomía y Salud, OMS, Washington, Estados Unidos.

PAGE Hans (2013). "Global Governance and Food Security as Global Public Good" en: Center on International Cooperation, Agosto, Nueva York, Estados Unidos. 
PIÑEIRO Carlos (2004). La Nación Sudamericana. Del imperativo histórico-cultural a la realización económico-política, № 1, Nuevo Hacer Grupo Editor Latinoamericano, Buenos Aires, Argentina.

RAVI Kanbur (2002). "Regional versus Internacional Financial Institutions", en:Estevadeordal, E., Frantz, B., Nguyen, T.R. , Regional Public Goods: From Theory to Practice, Banci Interamericano de Desarrollo y Banco asiático de Desarrollo,Washington, Estados Unidos.

RURAL DEVELOPMENT USDA (2010). Food expenditures by families and individuals as a share of disposable personal income, USDA, Washington, Estados Unidos.

SANDLER Todd, LOEHR William y CAULEY Jon (1978). "The Political Economy of Public Goods and International Cooperation", en: Monograph Series in World Affairs, vol. 15, № 3, Denver, Estados Unidos.

SEPÚLVEDA Isidro (2010). La creación de UNASUR en el marco de la seguridad y la defensa, № 29, Centro Superior de Estudios de la Defensa Nacional, Documentos de Seguridad y Defensa, España.

UE (2012). "The history of the CAP". Disponible:http://ec.europa.eu/agriculture/caphistory/index_en.htm (consulta realizada 23/02/2017)

UNASUR (2015). "Constitutive Treaty 2012". Disponible: http://www.unasursg.org/uploads/0c/c7/0cc721468628d65c3c510a577e54519d/Tratadoconstitutivo-english-version.pdf (consulta realizada 23/02/2017)

UNNEVEHR Laurian (2004). "Mad Cows and Bt Potatoes: Global Public Goods in the food system" en: American Journal of Agricultural Economics, vol. 86, № 5, Diciembre, Oxford, Inglaterra.

Economics, vol 37, Diciembre.

(2007). "Food safety as a global public good" en: Agricultural

USDA (2012a). "Budget summary and annual performance plan U.S. department of agriculture". Disponible: http://www.obpa.usda.gov/budsum/FY12budsum.pdf (consulta realizada 16/02/2017)

(2012b). "Overview".

http://www.usda.gov/wps/portal/usda/usdahome?navid=USDA150 (consulta realizada 16/02/2017).

(2015). "Budget summary and annual performance plan U.S. department of agriculture". Disponible: http://www.obpa.usda.gov/budsum/FY15budsum.pdf (consulta realizada 16/02/2017).

VÁZQUEZ Manuel (2013). "VII Reunión del Grupo de Trabajo 2025 Iniciativa América Latina y Caribe Sin Hambre (IALCSH)". Disponible: http://www.rlc.fao.org/es/iniciativa/seguimiento/gt2025/vii-reunion-del-gt2025/ (consulta realizada 22/02/2017). 

Año XXIII, N 36/Junio 2017, ISSN 2468-9912. DNDA: 5318259 en línea

Susana Herrero Olarte El rol de la sociedad civil en el reconocimiento de la seguridad alimentaria como bien público regional en Latinoamérica Págs. 67-85

(2014). “VIII Reunión del Grupo de Trabajo 2025 de la Iniciativa América Latina y Caribe sin Hambre (IALCSH)". Disponible: http://www.rlc.fao.org/es/iniciativa/seguimiento/gt2025/viii-reunion-del-gt2025/ (consulta realizada 22/02/2017).

Herrero Olarte Susana: Doctora en Economía Aplicada, 2014, Universidad Nacional Española. Especialista en crecimiento económico y desarrollo sostenible. Tesis defendida "Análisis crítico de la seguridad alimentaria como bien público global. El caso de las poblaciones marginadas y aisladas". Beca de excelencia MAE- AECID en 2011 y 2012. Máster "Gestión del Ciclo de Proyectos de Cooperación Internacional y Ayuda Humanitaria" en 2008 en la Universidad de Catalunya. Licenciatura en Ciencias Económicas en 2002 por la Universidad de Barcelona. Segundo Ciclo del Programa de Excelencia y Exclusividad Educativa y Académica E.U.S. de la Fundación Círculo de Economía y de la Fundación Bosch y Gimpera en la Universidad de Barcelona (UB). Directora del Centro de Investigaciones Económicas de la Universidad de las Américas (UDLA), Quito, Ecuador. Profesora titular de la UDLA e invitada en la Universidad de La Coruña y la Universidad Rey Juan Carlos I. Coordinadora regional para los países andinos y el Caribe de la Fundación CIDEAL de Investigación y Cooperación. Consultora para Unión Europea, Banco Mundial, etc. en el ámbito del desarrollo en México, Guatemala, Dominicana, Haití, Cuba, Colombia, Ecuador, Perú, Argentina, Malí, Níger y Vietnam.

Email: susana.herrero@udla.edu.ec / olartesusana@hotmail.com 